\begin{tabular}{c} 
International Journal of Engineering \& Technology, 7 (2)(2018) 750-754 \\
International Journal of Engineering \& Technology \\
SPC \\
Website: $\begin{array}{c}\text { ww. sciencepubco.com/index.php/IJET } \\
\text { doi: } 10.14419 / \text { ijet.v7i2.11527 } \\
\text { Research paper }\end{array}$ \\
\hline
\end{tabular}

\title{
Unsteady solute dispersion in blood rheology with reversible phase exchange at the artery wall
}

\author{
D. S. Sankar ${ }^{1 *}$, Nurul Aini Jaafar ${ }^{2}$, Yazariah Mohd Yatim ${ }^{2}$ \\ ${ }^{1}$ School of Applied Sciences and Mathematics, Universiti Teknologi Brunei, Gadong BE1410, Brunei Darussalam \\ ${ }^{2}$ School of Mathematical Sciences, Universiti Sains Malaysia, 11800 Penang, Malaysia \\ *Corresponding author E-mail: duraisamy.sankar@utb.edu.bn
}

\begin{abstract}
The effect of reversible phase exchange between the flowing fluid and wall tissues of arteries in the unsteady dispersion of solute in blood flow through a narrow artery is analysed mathematically, modelling the blood as Casson fluid. The resulting convective diffusion equation along with the initial and boundary conditions is solved analytically using the derivative series expansion method. The expressions for the negative asymptotic phase exchange, negative asymptotic convection, longitudinal diffusion coefficient and mean concentration are obtained. It is noted that when the solute disperses in blood flow through a narrow artery, the negative exchange coefficient, the negative convection coefficient increase and the longitudinal diffusion coefficient decreases with the increase of the Damköhler number and partition coefficient.
\end{abstract}

Keywords: Blood Flow; Narrow Artery; Casson Fluid; Unsteady Solute Dispersion; Reversible Phase Exchange.

\section{Introduction}

Taylor [1] was the first researcher who theoretically analysed the solute dispersion in a laminar flow through a circular pipe. Several researchers [2-5] extended his study by incorporating various physical parameters. Gill and Sankarasubramanian [5] introduced generalized dispersion model (GDM) to demonstrate the whole dispersion process. Reversible phase exchange is an exchange process between the fluid and thin tissue layer at the vessel wall which is an important physical phenomenon. The studies pertaining to reversible reactions were taken up by several researchers [6], [7]. It is well known that the phenomena of the solute dispersion in a solvent flow in the presence of phase exchange between the flowing fluid and vessel wall has several physical applications, one such application is the dispersion of medicines in blood flow in arteries. The previous literatures used Newtonian fluid modelling blood when it flows through larger arteries at high shear rates [8]. Casson fluid is a nonNewtonian fluid model which is suitable for representing blood when it flows through narrow arteries at low shear rates [9]. Hence, in this paper, we mathematically analyze the solute dispersion in blood flow through narrow arteries in the presence of phase exchange between the flowing fluid (blood miscible with solute) and the thin tissue layer at the wall of the artery, treating the blood as Casson fluid model.

\section{Mathematical formulation}

Consider the unsteady dispersion of solute in the axi-symmetric, steady, laminar and fully developed unidirectional flow of blood through a circular pipe (narrow artery), treating the blood as Casson fluid. The geometry of fluid flow through a circular pipe is shown in Fig. 1. Cylindrical polar coordinate system $(\bar{r}, \bar{\psi}, \bar{z})$ is used to analyze the flow in a circular pipe, where $\bar{\psi}$ is the azimuthal angle, $\overline{\mathrm{r}}$ and $\bar{z}$ are the coordinates in the radial and axial directions, respectively.

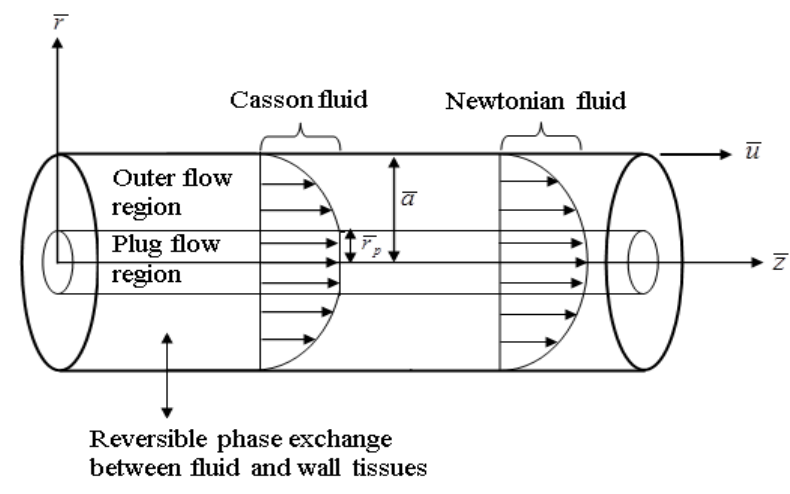

Fig. 1: The Geometry of Fluid Flow in A Circular Pipe.

\subsection{Governing equations}

The unsteady convective-diffusion equation for flow in a circular pipe is given by [9]

$$
\frac{\partial \bar{C}}{\partial \bar{t}}+\bar{u} \frac{\partial \bar{C}}{\partial \bar{z}}=\bar{D}_{m}\left(\frac{1}{\bar{r}} \frac{\partial}{\partial \bar{r}}\left(\bar{r} \frac{\partial}{\partial \bar{r}}\right)+\frac{\partial^{2}}{\partial \bar{z}^{2}}\right) \bar{C}
$$

where $C(\bar{r}, \bar{z}, \bar{t})$ is the concentration of the solute and $\bar{t}$ is the time variable with the non-uniform initial condition [10]

$$
\overline{\mathrm{C}}(\overline{\mathrm{r}}, \overline{\mathrm{z}}, 0)=\left\{\begin{array}{c}
\overline{\mathrm{C}}_{0} \overline{\mathrm{Y}}_{1}(\overline{\mathrm{z}}) \overline{\mathrm{Y}}_{2}(\overline{\mathrm{r}}) \text { if }|\overline{\mathrm{z}}| \leq \overline{\mathrm{z}}_{\mathrm{s}} / 2 \\
0 \text { if }|\overline{\mathrm{z}}|>\overline{\mathrm{z}}_{\mathrm{s}} / 2
\end{array}\right.
$$


Where $\overline{\mathrm{C}}_{0}$ is the reference concentration, $\overline{\mathrm{Y}}_{1}(\overline{\mathrm{z}})$ and $\overline{\mathrm{Y}}_{2}(\overline{\mathrm{z}})$ are the separable function of $\overline{\mathrm{z}}$ and $\overline{\mathrm{r}}$ as given by

$$
\begin{aligned}
& \bar{Y}_{1}(\overline{\mathrm{z}})==\bar{a} \bar{\delta}(\bar{z}) / \bar{d}^{2}, \\
& \bar{Y}_{2}(\bar{r})=\left\{\begin{array}{lll}
1 & \text { if } & 0 \leq \bar{r} \leq \bar{a} \bar{d}, \\
0 & \text { if } & \bar{a} \bar{d}<\bar{r} \leq \bar{a},
\end{array}\right.
\end{aligned}
$$

Where $\bar{\delta}(\overline{\mathrm{z}})$ is the Dirac delta function and $\overline{\mathrm{d}}$ is the cross-section of circle of radius concentric with the pipe. The boundary conditions of (1) with the reaction at the wall are

$$
\begin{aligned}
& -\bar{D}_{m} \partial \bar{C} / \partial \bar{r}=\partial \overline{C_{s}} / \partial \bar{t}=\bar{k}\left(\bar{\sigma} \bar{C}-\bar{C}_{s}\right) \text { at } \bar{r}=\bar{a}, \\
& \partial \bar{C} / \partial \bar{r}=0 \text { at } \bar{r}=0 \\
& \bar{C}(\bar{r}, \infty, \bar{t})=[\partial \bar{C} / \partial \bar{z}](\bar{r}, \infty, \bar{t})=0 \\
& \bar{C}(0, \bar{z}, \bar{t})=\text { finite }
\end{aligned}
$$

Where $\overline{\mathrm{k}}$ is the reaction rate and $\bar{\sigma}=\left(\overline{\mathrm{C}}_{\mathrm{s}} / \overline{\mathrm{C}}\right)_{\text {equilibrium }}$ is the equilibrium partition coefficient between the blood and wall.

\subsection{Non-dimensional variables}

Let us introduce the following non-dimensional variables:

$C=\bar{C} / \bar{C}_{0}, u=\bar{u} / \bar{u}_{0}, r=\bar{r} / \bar{a}, r_{p}=\bar{r}_{p} / \bar{a}, z=\bar{D}_{m} \bar{z} / \bar{a}^{2} \bar{u}_{0}, t=\bar{D}_{m} \bar{t} / \bar{a}^{2}$,

$\tau=\bar{\tau} /\left(\bar{\eta}_{H} \bar{u}_{0} / \bar{a}\right)^{1 / n}, \tau_{y}=\bar{\tau}_{y} /\left(\bar{\eta}_{H} \bar{u}_{0} / \bar{a}\right)^{1 / n}, D a=\bar{k} \bar{a}^{2} / \bar{D}_{m}, \sigma=\bar{\sigma} / \bar{a}$,

$Y_{1}=\overline{Y_{1}}, Y_{2}=\overline{Y_{2}}, d=\bar{d}, \delta=\bar{\delta}$,

Where $\overline{\mathrm{r}}_{\mathrm{p}}$ is plug core radius. The non-dimensional form of Eq. (1) is

$$
\frac{\partial C}{\partial t}+u \frac{\partial C}{\partial z}=\left(\frac{1}{r} \frac{\partial}{\partial r}\left(r \frac{\partial}{\partial r}\right)+\frac{1}{P e^{2}} \frac{\partial^{2}}{\partial z^{2}}\right) C .
$$

The non-dimensional form of Eqs. (2), (5)-(8) are

$$
\begin{aligned}
& C(r, z, 0)= \begin{cases}Y_{1}(z) Y_{2}(r) \text { if }|z| \leq z_{s} / 2, \\
0 & \text { if }|z|>z_{s} / 2,\end{cases} \\
& Y_{1}(z)=\delta(z) / d^{2} P e, \\
& Y_{2}(r)=\left\{\begin{array}{lll}
1 & \text { if } & 0<r \leq d, \\
0 & \text { if } & d<r \leq 1,
\end{array}\right. \\
& -\frac{\partial C}{\partial r}(1, z, t)=\frac{\partial C_{s}(z, t)}{\partial t}=D a\left[\sigma C(1, z, t)-C_{s}(z, t)\right], \\
& \partial C / \partial r=0 \text { at } r=0, \\
& C(r, \infty, t)=\frac{\partial C}{\partial z}(r, \infty, t)=0,
\end{aligned}
$$

\section{Method of solution}

\subsection{Dispersion functions and coefficients}

The solution of (10) subject to the (11) and (14)-(17) is [15]

$C\left(r, z_{1}, t\right)=\sum_{i=0}^{\infty} f_{i}(r, t) \frac{\partial^{i} C_{m}\left(z_{1}, t\right)}{\partial z_{1}^{i}}$,

Where $\mathrm{f}_{\mathrm{i}}$ is the dispersion function, $\mathrm{z}_{1}$ is a new axial coordinate and $\mathrm{C}_{\mathrm{m}}$ is the mean concentration of solute given by

$$
C_{m}\left(z_{1}, t\right)=2 \int_{0}^{1} C\left(r, z_{1}, t\right) r d r .
$$

We expand the mean concentration as [6]

$\frac{\partial C_{m}}{\partial t}\left(z_{1}, t\right)=\sum_{i=0}^{\infty} K_{i}(t) \frac{\partial^{i} C_{m}}{\partial z_{1}^{i}}\left(z_{1}, t\right)$

Where $\mathrm{K}_{\mathrm{i}}$ are the dispersion coefficients and are given by

$$
K_{i}(t)=\frac{\delta_{i 2}}{P e^{2}}+2 \frac{\partial f_{i}}{\partial r}(1, t)-2 \int_{0}^{1} f_{i-1}(r, t) u(r, t) r d r, i=0,1,2, \ldots
$$

With the Kronecker delta $\delta_{i j}$. Substituting (18) and (20) into (10) and grouping the coefficients of $\partial^{i} C_{m} / \partial z_{1}^{i}$, we have

$\frac{\partial f_{i}}{\partial t}(r, t)=\frac{1}{r} \frac{\partial}{\partial r}\left(r \frac{\partial}{\partial r}\right) f_{i}(r, t)-u(r, t) f_{i-1}(r, t)+\frac{f_{i-2}}{P e^{2}}(r, t)$

$-\sum_{j=0}^{i} K_{j}(t) f_{i-j}(r, t), i=0,1,2, \ldots$

Where $\mathrm{f}_{-1}=\mathrm{f}_{-2}=0 . \operatorname{In}(22)$, to find $\mathrm{K}_{\mathrm{i}}$, we need $\mathrm{f}_{\mathrm{i}}$. Use of Eq. (18) in Eqs. (11), (14), (17) and (19), (22) yields the respective initial, boundary and solvability conditions

$f_{i}(r, 0)=0, \quad i=1,2, \ldots$

$\left[\partial f_{i} / \partial r\right](1, t)=-\beta f_{i}(1, t)-\operatorname{Da\sigma f}_{i}(1, t), \quad i=0,1,2, \ldots$,

$f_{i}(0, t)=$ finite $, \quad i=0,1,2, \ldots$

$\int_{a}^{1} f_{i}(r, t) r d r=\delta_{i 0} / 2$

Setting $i=0$ in Eq. (22), we get the differential equation for $f_{0}$ as

$\frac{\partial f_{0}}{\partial t}(r, t)=\frac{1}{r} \frac{\partial}{\partial r}\left(r \frac{\partial f_{0}}{\partial r}(r, t)\right)-f_{0}(r, t) K_{0}(t)$.

With the initial and the solvability conditions

$f_{0}(r, 0)=Y_{2}(r) / 2 \int_{0}^{1} Y_{2}(r) r d r$

$$
\int_{0}^{1} f_{0}(r, t) r d r=1 / 2 \text {. }
$$

Solving Eq. (27) using the variable separable method subject to Eqs. (24), (25), (28) and (29), the solution of $f_{0}(r, t)$ is found as

$$
f_{0}(r, t)=\frac{\sum_{s=0}^{\infty} A_{s} J_{0}\left(\mu_{s} r\right) e^{-\mu_{t}^{2} t}}{2 \sum_{s=0}^{\infty}\left(\frac{A_{s}}{\mu_{s}}\right) J_{1}\left(\mu_{s}\right) e^{-\mu_{i}^{2} t}} .
$$

Where $\mu_{s}$ is the root of the transcendental equation

$$
\mu_{s} J_{1}\left(\mu_{s}\right)=D a \sigma J_{0}\left(\mu_{s}\right), \quad s=0,1,2, \ldots
$$




$$
A_{s}=\frac{\mu_{s}^{2} \int_{0}^{1} J_{0}\left(\mu_{s} r\right) Y_{2}(r) r d r}{J_{0}^{2}\left(\mu_{s}\right)\left\{\mu_{s}^{2}+(D a \sigma)^{2}\right\} \int_{0}^{1} Y_{2}(r) r d r} .
$$

As $f_{0}(r, t)$ is known, $K_{0}(t)$ is obtained from Eq. (21) as below:

$$
K_{0}(t)=2 \frac{\partial f_{0}}{\partial r}(1, t)=-\frac{\sum_{s=0}^{\infty} A_{s} \mu_{s} J_{1}\left(\mu_{s}\right) e^{-\mu_{s}^{2} t}}{\sum_{s=0}^{\infty}\left(\frac{A_{s}}{\mu_{s}}\right) J_{1}\left(\mu_{s}\right) e^{-\mu_{s}^{2} t}} .
$$

Since the computations of the higher order $f_{1}, f_{2}, \ldots$ and $K_{1}, K_{2}, \ldots$ have difficult integral equations, the asymptotic computation has been used to give beneficial physical insight into the nature of the problem [6]. At large time, expanding Eq. (30) and ignoring the negligibly small terms in Eq. (30) yields

$$
f_{0}(r)=\mu_{0} J_{0}\left(\mu_{0} r\right) / 2 J_{1}\left(\mu_{0}\right) \text {. }
$$

Applying Eq. (32) in Eq. (33), $\mathrm{K}_{0}$ at large time is obtained as

$$
K_{0}=-\mu_{0}^{2},
$$

Where $\mu_{0}$ is the first root of Eq. (31). Using Eq. (35) in Eq. (22), we obtain the following differential equation at large time for $\mathrm{f}_{\mathrm{i}}(\mathrm{r})$ :

$$
\begin{aligned}
& \frac{1}{r} \frac{d}{d r}\left(r \frac{\partial f_{i}}{\partial r}(r)\right)+\mu_{0}^{2} f_{i}(r)=u(r) f_{i-1}(r) \\
& -\frac{f_{i-2}}{P e^{2}}(r)+\sum_{j=1}^{i} K_{j} f_{i-j}(r)
\end{aligned}
$$

And using Eq. (35) in Eqs. (24), (25) and (26), we obtain the following conditions:

$$
\begin{aligned}
& {[d f / d r](1)=-\operatorname{Da\sigma f}_{i}(1), i=1,2,3, \ldots,} \\
& f_{i}(0)=\text { finite } \\
& \int_{a}^{1} f_{i}(r) r d r=0, \quad i=1,2,3, \ldots
\end{aligned}
$$

Note that $\mathrm{K}_{\mathrm{j}}$ 's in Eq. (36) are the constant steady-state $(t \rightarrow \infty)$ values. Multiplying both sides of Eq. (21) by $\mathrm{rJ}_{0}\left(\mu_{0} \mathrm{r}\right)$ and then integrating it with respect to $r$ and applying the orthogonal property of Bessel function and using Eqs. (34) and (39), we get $\mathrm{K}_{\mathrm{i}}$ as below:

$$
K_{i}=\frac{\int_{0}^{1} r J_{0}\left(\mu_{0} r\right)\left[\frac{1}{P e^{2}} f_{i-2}(r)-u(r) f_{i-1}(r)-\sum_{j=1}^{i-1} K_{j} f_{i-j}(r)\right] d r}{\int_{0}^{1} f_{0}(r) r J_{0}\left(\mu_{0} r\right) d r} .
$$

In order to get the solution of longitudinal convection coefficient $\mathrm{K}_{1}$, we apply $\mathrm{i}=1$ in (34) and using Eq. (40), we get

$$
K_{1}=-\frac{-2 \mu_{0}^{2} \int_{0}^{1} u(r) r J_{0}^{2}\left(\mu_{0} r\right) d r}{\left\{\mu_{0}^{2}+(D a \sigma)^{2}\right\} J_{0}^{2}\left(\mu_{0}\right)} .
$$

Thus, Eq. (36) is reduced to

$$
\frac{1}{r} \frac{d}{d r}\left(r \frac{\partial f_{1}}{\partial r}(r)\right)+\mu_{0}^{2} f_{1}(r)=u(r) f_{0}(r)+K_{0} f_{0}(r)
$$

And the respective boundary and solvability conditions reduce to

$\left[d f_{1} / d r\right](1)=-D a \sigma f_{1}(1)$

$f_{1}(0)=$ finite $\int_{0}^{1} f_{1}(r) r d r=0$

Using Eqs. (41), (43)-(45), the solution of Eq. (42) is obtained as

$f_{1}(r)=\sum_{s=1}^{\infty} B_{s}\left[-\frac{\mu_{0} J_{1}\left(\mu_{s}\right) J_{0}\left(\mu_{0} r\right)}{\mu_{s} J_{1}\left(\mu_{0}\right)}+J_{0}\left(\mu_{s} r\right)\right]$.

Using Bessel properties, we get $\mathrm{B}_{\mathrm{s}}$, as

$B_{s}=\frac{2 \mu_{s}^{2} \int_{0}^{1}\left[u(r)+K_{1}\right] f_{0}(r) J_{0}\left(\mu_{s} r\right) r d r}{\left(\mu_{0}^{2}-\mu_{s}^{2}\right)\left\{\mu_{s}^{2}+(\beta+\operatorname{Da} \sigma)^{2}\right\} J_{0}^{2}\left(\mu_{s}\right)}$.

Once $\mathrm{f}_{1}$ is known, the longitudinal diffusion coefficient $\mathrm{K}_{2}$ can be obtained as below by setting $\mathrm{i}=2$ in Eq. (40):

$K_{2}=\frac{1}{P e^{2}}-\frac{4 \mu_{0} J_{1}\left(\mu_{0}\right)}{\left\{\mu_{0}^{2}+(\beta+\mathrm{Da} \sigma)^{2}\right\} J_{0}^{2}\left(\mu_{0}\right)} \times$

$\int_{0}^{1}\left[u(r)+K_{1}\right] f_{1}(r) J_{0}\left(\mu_{0} r\right) r d r$

\subsection{Mean concentration}

Expanding Eq. (20) and then neglecting the terms $\mathrm{K}_{3}(\mathrm{t}), \mathrm{K}_{4}(\mathrm{t}), \ldots$ (since the magnitude of these terms are negligibly small), one can obtain

$\frac{\partial C_{m}}{\partial t}=K_{0} C_{m}+K_{1} \frac{\partial C_{m}}{\partial z_{1}}+K_{2} \frac{\partial^{2} C_{m}}{\partial z_{1}^{2}}$

Substituting Eqs. (11) and (16) into (19), we get the following initial and boundary conditions:

$$
\begin{aligned}
& C_{m}\left(z_{1}, 0\right)=2 Y_{1}\left(z_{1}\right) \int_{0}^{1} Y_{2}(r) r d r, \\
& C_{m}(\infty, t)=\left[\partial C_{m} / \partial z_{1}\right](\infty, t)=0 .
\end{aligned}
$$

The solution for $C_{m}(z, t)$ is obtained as below by solving Eq. (49) subject to the conditions (50) and (51) and using Fourier Transform (FT) method:

$C_{m}\left(z_{1}, t\right)=\frac{1}{2 P e \sqrt{\pi \phi}} e^{\left(5-\frac{z_{1}^{2}}{4 \phi}\right)}$,

Where

$$
\zeta(t)=\int_{0}^{t} K_{0}(t) d t
$$

$z_{1}(z, t)=z+\int_{0}^{t} K_{1}(t) d t$

$\phi(t)=\int_{0}^{t} K_{2}(t) d t$

\section{Results and discussions}

The objective of this paper is to discuss the effects of various parameters on the negative asymptotic phase exchange, negative asymptotic convection, longitudinal diffusion coefficients and mean concentration

\subsection{Negative exchange coefficient}

The variation of negative asymptotic exchange coefficient $\left(-K_{0}\right)$ with Damköhler number $(D a)$ for different values of partition coefficient $\sigma$ is shown in Fig. 2. It is noted that $\left(-K_{0}\right)$ increases very 
rapidly with the increase of $D a$ from 0 to 10 and then it increases very slowly (almost constant) from 10 to 100 . It is also observed that $\left(-K_{0}\right)$ increases when $\sigma$ increases due to the increasing of the molecules reaction at the wall, thus, the solute exchange becomes faster. At quite large value of $D a$ and $\sigma,\left(-K_{0}\right)$ becomes effective when a high amount of the solute has been exchanged to the wall tissues of blood vessels and less amount of solute at the central region.

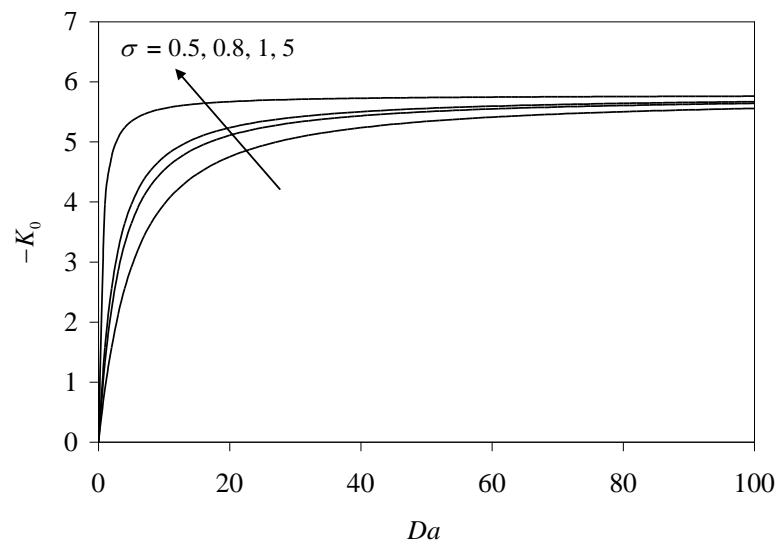

Fig. 2: Variation of $\left(-\mathrm{K}_{0}\right)$ with Da for different values of $\sigma$.

\subsection{Negative asymptotic convection coefficient}

The variation of negative asymptotic convection coefficient $\left(-K_{1}\right)$ with Damköhler number $D a$ for different values of partition coefficient $\sigma$ and yield stress $r_{p}$ is depicted in Fig. 3. It is noticed that ($K_{1}$ ) increases when $D a$ and $\sigma$ increase and it tends to be a constant when the value of $D a$ is large $(D a>10)$. It is also clear that $\left(-K_{1}\right)$ decreases when $r_{p}$ increases because when $r_{p}$ increases, the accumulation of red blood cells at the centre increase and thus, the velocity tends to decrease and it slows down the convection process. The plot of $\left(-K_{1}\right)$ for Newtonian fluid $\left(r_{p}=0\right)$ without the phase exchange $(\sigma=0)$ is in good agreement with the corresponding plot given in Gill and Sankarasubramanain [5].

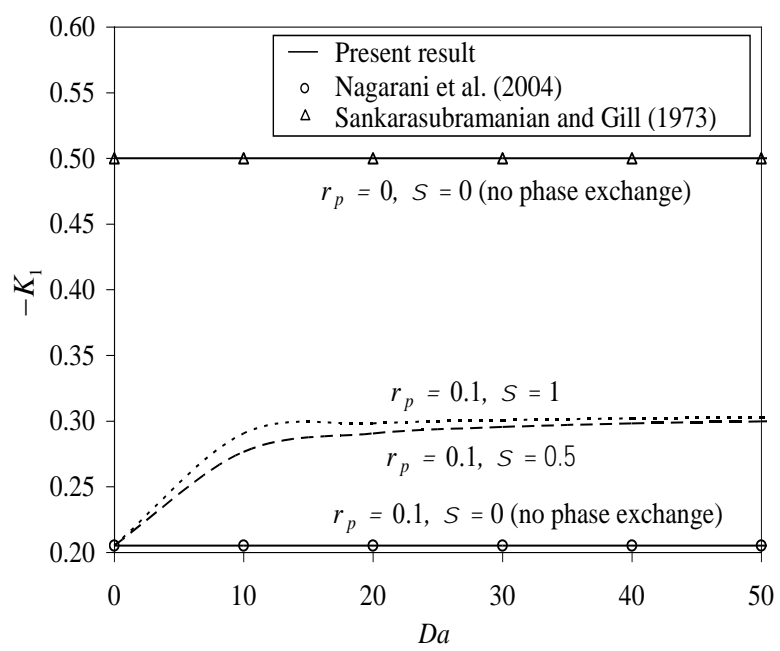

Fig. 3: Variation Of $\left(-\mathrm{K}_{1}\right)$ With Da for Various Values of $\sigma$ and $\mathrm{R}_{\mathrm{p}}$.

\subsection{Longitudinal diffusion coefficient}

Fig. 4 shows the variation of the longitudinal diffusion coefficient $\left(K_{2}-1 / P e^{2}\right) \times 103$ with Damköhler number $D a$ for different values of partition coefficient $\sigma$. It is seen that the $\left(K_{2}-1 / P e^{2}\right)$ x 103 decreases very rapidly with the increase of $D a$ from 0 to 20 and then it decreases slowly from when $D a$ increases from 20 to 100 . It is also found that the $\left(K_{2}-1 / P e^{2}\right) \times 103$ decreases with the increase of $\sigma$. It is of interest to note that the plot of $\left(K_{2}-1 / P e^{2}\right) \times 10^{3}$ for Newtonian with $\sigma=0$ is in good agreement with the corresponding plot in Gill and Sankarasubramanian [5].

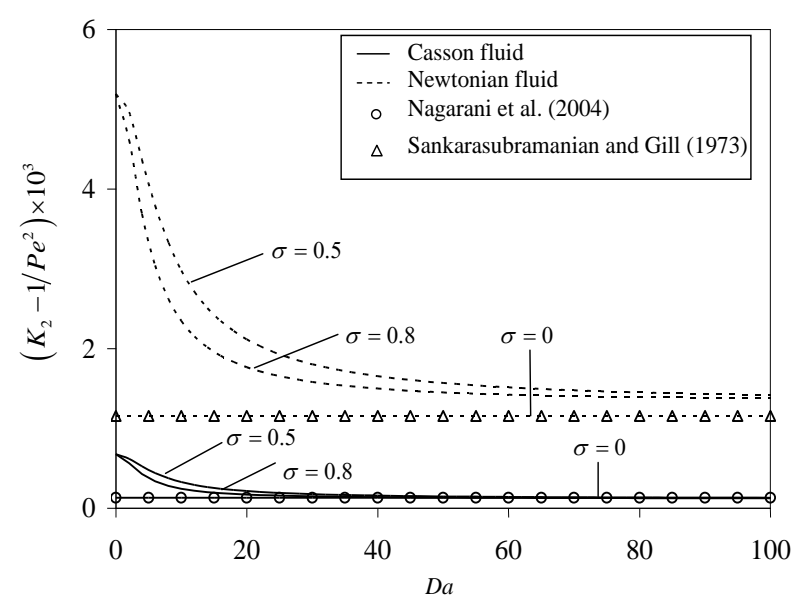

Fig. 4: Variation of $\left(\mathrm{K}_{2}-1 / \mathrm{Pe}^{2}\right) 10^{3}$ with Da for Different Values of $\sigma$ and $\mathrm{R}_{\mathrm{p}}$.

\subsection{Mean concentration of solute}

Fig. 5 depicts the variation of $C_{m} \times P e$ with time $t$ at $z=0.5$ for different values of $D a, \sigma$ and $r_{p}$. In an early period of time, $C_{m} \times P e$ increases rapidly and reaches the maximum value at the middle of the period and then it falls suddenly. The peaks in the graph show an effective $C_{m} \times P e$ and it helps us to predict the time to achieve effective $C_{m} \times P e$ in Casson fluid as 0.3 and Newtonian fluid as 0.1 .

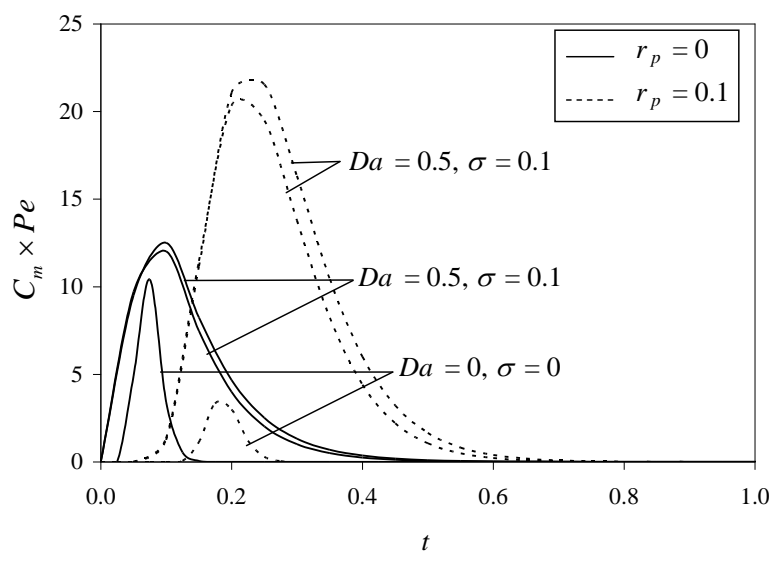

Fig. 5: Variation of $C_{m} \times P e$ with $D a$ for Different Values of $\sigma$ And $R_{p}$.

\section{Conclusions}

This study investigates the effects of yield stress and reversible reactions at the wall on the phase exchange, convection, longitudinal diffusion coefficients and mean concentration of the solute. The major findings of this study are summarised below:

- The negative exchange coefficient, the negative convective coefficient increase and the longitudinal diffusion coefficient decreases with the increase of the Damköhler number and partition coefficient.

- The negative exchange coefficient, the negative convective coefficient and the longitudinal diffusion coefficient decrease with the increase of yield stress.

In view of the results obtained, this study may be considered as an improvement in the studies of solutes dispersion in blood flow through arteries with reversible reaction at the wall of the artery.

\section{Acknowledgements}

This research work was supported by the Research University Grant of Universiti Sains Malaysia, Malaysia (RU Grant ref. no: 1001/PMATHS/811177). 


\section{References}

[1] Taylor G. (1953). "Dispersion of soluble matter in solvent flowing slowly through a tube", Proc. R. Soc. A Math. Phys. Eng. Sci. 219 (1137): 186-203. https://doi.org/10.1098/rspa.1953.0139.

[2] Aris R. (1956). "On the dispersion of a solute in a fluid flowing through a tub", Proc. R. Soc. A Math. Phys. Eng. Sci. 235 (1200): 66-77. https://doi.org/10.1098/rspa.1956.0065.

[3] Bailey H. R. and Gogarty W. B. (1962). "Numerical and experimental results on the dispersion of a solute in a fluid in laminar flow through a tube", Proc. R. Soc. A Math. Phys. Eng. Sci. 333 (1592): 99-114. https://doi.org/10.1098/rspa.1962.0182.

[4] Ananthakrishnan V., Gill W. N. and Barduhn A. J. (1965). "Laminar dispersion in capillaries", A.I.Ch. E. J. 11(6): 1063-1072. https://doi.org/10.1002/aic.690110620.

[5] Sankarasubramanian R. and Gill W. N. (1973). "Unsteady convective diffusion with interphase mass transfer", Proc. R. Soc. A Math Phys. Eng. Sci. 333(1592): 115-132. https://doi.org/10.1098/rspa.1973.0051.

[6] Ng C. O. (2006). "Dispersion in open-channel flow subject to the processes of sorptive exchange on the bottom and air-water exchange on the free surface". J. Hydrodyn. 18 (3): 57-64. https://doi.org/10.1016/S1001-6058(06)60031-0.

[7] Zhang D., Lu Z., Liu Y. and Ng C. (2009). "Numerical simulation of the dispersion in oscillating flows with reversible and irreversible wall reactions", J. Hydrodyn. 21(4): 482-490. https://doi.org/10.1016/S1001-6058(08)60174-2.

[8] Chiu N. G. and YIP T. L. (2001). "Effects of kinetic absorptive exchange on solute transport in open channel flow", J. Fluid Mech. 446: 321-345.

[9] Scott Blair G. W. (1966). "The success of Casson's equation”, Rheol Acta 5(3): 184-187. https://doi.org/10.1007/BF01982424.

[10] Gill W. N. and Sankarasubramanian R. (1971). "Dispersion of a nonuniform slug in time-dependent flow", Proc. R. Soc. London A, 322: 101-117. https://doi.org/10.1098/rspa.1971.0057. 